\title{
Role of Anti-Tissue Transglutaminase IgA Antibody in Aborted Women Infected with Toxoplasma Gondii in Association with Latent Coeliac Disease
}

\author{
Shukryia Shadhan Chyad \\ College of Health and Medical Techniques, Al-Furat Al-Awsat Technical University, 54003 Al-Kufa, Iraq. \\ $\triangle$ Corresponding author. E-mail: kuh.shu@atu.edu.iq
}

Received: Sep. 28, 2017; Accepted: Dec. 5, 2017; Published: Dec. 25, 2017.

Citation: Shukryia Shadhan Chyad, Role of Anti-Tissue Transglutaminase IgA Antibody in Aborted Women Infected with Toxoplasma Gondii in Association with Latent Coeliac Disease. Nano Biomed. Eng., 2017, 9(4): 306-3।3.

DOI: $10.5101 /$ nbe.v9i4.p306-313.

\begin{abstract}
Toxoplasma gondii is an obligate intracellular protozoan parasite that is capable of infecting a variety of intermediate hosts including humans. Infected definitive hosts (cats) shed oocysts in feces that rapidly mature in the soil and become infectious. Vertical transmission of the parasite through the placenta can also occur, leading to congenital toxoplasmosis. Following primary infection, Toxoplasma gondii can remain latent for the life of the host; the risk for reactivation is highest among immunosuppressed individuals. The current study was planned by using 125 pregnant women with single or multiple abortion from different private clinics and hospitals in Babylon province, as well as a control group of 215 pregnant women without history of abortion and 15 apparently healthy women without pregnancy. For all patients and controls, screening tests for anti-Toxoplasma (antiToxo) antibody (IgM and $\mathrm{IgG}$ ) and anti-transglutaminase/Immunoglobulin A (AtTg/IgA) antibody level were done by using ELISA technique in the laboratory of Babylon GIT Center during the period from August 2016 to June 2017. Descriptive and inferential statistical analysis was used to analyze the data. The result revealed that IgG was only indicative of previous exposure to Toxoplasma (recent or past IgG. Women aged 24-35 years were more susceptible to abortion than the other age groups, mostly during the first trimester of pregnancy. There was a relationship between aborted women having toxoplasma infection with AtTg antibody, which might suggest a latent coeliac disease (asymptomatic) that was associated with pregnancy and led to spontaneous abortion. The AtTg IgA had significant negative correlation with age group, time of abortion and abortion number. This result might indicate that AtTg IgA spontaneously increased with time in relation to patient ages as well as specific conditions of aborted women such as time and number of abortion.
\end{abstract}

Keywords: Transglutaminase IgA antibody; Aborted women; Toxoplasma gondii; Coeliac disease

\section{Introduction}

Toxoplasma gondii is an obligate intracellular protozoan parasite that is capable of infecting a variety of intermediate hosts including humans. Infected definitive hosts (cats) shed oocysts in feces that rapidly mature in the soil and become infectious [1]. Toxoplasmosis is acquired by humans through ingestion of food or water contaminated with cat feces or through eating undercooked meat containing viable oocysts. Vertical transmission of the parasite through the placenta can also occur, leading to 
congenital toxoplasmosis. Following primary infection, Toxoplasma gondii can remain latent for the life of the host; the risk for reactivation is highest among immunosuppressed individuals.

Seri prevalence studies performed in the United States indicated that approximately $9 \%$ to $11 \%$ of individuals between the ages of 6 and 49 years had antibodies to Toxoplasma gondii [2]. Subclinical infection may result in premature delivery and subsequent neurologic, intellectual and audio logic defects. Active Toxoplasmosis is suggested by the presence of IgM antibodies; however, elevated antiIgM titers are often absent in immunocompromised patients. In addition, elevated IgM can persist from an acute infection that may have occurred as long ago as one year.

$\operatorname{IgG}$ is only indicative of previous exposure to Toxoplasma (recent or past). A single positive Toxoplasma IgG result should not be used to diagnose the recent infection. Seroconversion from negative to positive $\operatorname{IgG}$ is indicative of recent Toxoplasma gondii infection. A suspected diagnosis of acute Toxoplasmosis should be confirmed by detection of Toxoplasma gondii DNA by polymerase chain reaction (PCR) analysis of cerebrospinal fluid or amniotic fluid specimens (PTOX / Toxoplasma gondii, Molecular Detection, PCR). Sera drawn very early during the acute stage of infection may have Toxoplasma IgG levels $<9 \mathrm{IU} / \mathrm{mL}[1]$.

The frequency of dead children birth was about the same: $2.3 \%$ and $1.9 \%$ respectively $(p>0.05)$. In $43 \%$ of women after 6-8 months of strict adherence to a gluten-free diet (GFD), amenorrhea had disappeared and there were regular menses. Three women of childbearing age strictly abided the GFD and had a history of repeated spontaneous abortion during the year managed to get pregnant and give birth to a healthy full-term baby. Reproductive disorders in women with celiac disease (CD) are significantly more likely than at women with functional bowel disease. One of the reasons of reproductive disorders in patients with CD may be the malabsorption of necessary nutrients in the small intestine. The presence of reproductive disorders should be considered as a risk factor for $\mathrm{CD}$, and so these women should be screened for $\mathrm{CD}[3]$.

$\mathrm{CD}$ is an immune-mediated small bowel disorder that develops in genetically susceptible individuals upon exposure to dietary gluten. CD could have extra-intestinal manifestations that affect women's reproductive health. The HLA-DQ2 and DQ8 haplotypes are expressed on the surface of antigenpresenting cells in the gut lamina propria and bind activated gliadin peptides, eliciting an inflammatory reaction. This inflammatory state leads to changes in the small bowel mucosa architecture including increased infiltration of lymphocytes into the epithelial cells, villous atrophy and crypt distortion. $\mathrm{CD}$ is associated with a number of extra intestinal manifestations, and resultant morbidity and mortality [4]. An association between $C D$ and reproductive abnormalities was first made in 1970 when Morris et al. described three patients with untreated CD and infertility, all of whom became pregnant after initiating a GFD. However, since this case report, the literature addressing complications of CD in women, especifically the rate of infertility, length of fertile life span, perinatal complications and adverse pregnancy outcomes, has been inconsistent [5].

Women with CD have increased spontaneous abortions, preterm delivery, and fewer successful pregnancies. With the increase in the prevalence of $\mathrm{CD}$, over the last several decades and the advent of celiac centers in the U.S. and across the world, the fertility experience of these patients is an important aspect of women's health that needs increased awareness among patients and physicians. Though there are conflicting data regarding the relative risk of infertility and other reproductive complications, undiagnosed CD should be considered as an etiology in patients with recurrent complications of pregnancy, and these women should be screened for serologic markers. Women's health specialists need to be aware of the pregnancy complication faced by women with untreated $\mathrm{CD}$ and have a low threshold for testing high-risk patients [6].

The seroprevalence of transglutaminase IgA was $6.70 \%$ in the group with recurrent abortion, $5.70 \%$ in the group with stillbirth, $5.65 \%$ in the group with infertility, $9.33 \%$ in the group with intrauterine growth restriction, and $1.30 \%$ in the control group. Rates of previous preterm births, low birthweight infants and cesarean section were higher in seropositive women compared with seronegative subjects. Women having poor reproductive performance had subclinical CD. The serology for $\mathrm{CD}$ can be considered in idiopathic cases [7].

Spontaneous abortion, is the loss of pregnancy without intervention before 20 weeks of gestation, 
affecting up to $20 \%$ of recognized pregnancies. Spontaneous abortion can be subdivided into threatened abortion, inevitable abortion, incomplete abortion, missed abortion, septic abortion, complete abortion and recurrent spontaneous abortion. Many pregnancies are lost spontaneously before a woman recognizes that she is pregnant, and the clinical signs of miscarriage are mistaken for a heavy or late menses. No clear association has been found between the influence of stress and early pregnancy loss. Sexual activity also does not elevate the risk in women with uncomplicated pregnancies. The chronic maternal diseases are poorly controlled diabetes, $\mathrm{CD}$, autoimmune diseases (particularly antiphospholipid antibody syndrome),and the maternal infections are bacterial vaginosis; mycoplasmosis, herpes simplex virus, toxoplasmosis, listeriosis, chlamydia, human immunodeficiency virus, syphilis, parvovirus B19, malaria, gonorrhea, rubella, cytomegalovirus [8].

Patients presenting silent $\mathrm{CD}$, without gastrointestinal (GI) symptoms, may have short stature or neurologic symptoms and possible reproductive disorders, including delayed menarche, menstrual disturbances, infertility and recurrent miscarriage. $\mathrm{CD}$ has also been associated with chronic pelvic pain, including dysmenorrhea and deep dyspareunia. The underlying injury involves an inflammatory reaction, primarily in the duodenum and jejunum, mediated by gliadin reactive $\mathrm{CD} 4+\mathrm{T}$ cells in the lamina propria [9]. These cells are bound to gliadin, a primary wheat protein, and produce inflammatory cytokines, especially interferon $\gamma$ [7]. The immunogenicity of gliadin is enhanced by the enzyme tissue transglutaminase, which dimidiates the gliadin peptides and serves as a marker for $\mathrm{CD}$. The mechanisms by which $\mathrm{CD}$ affects reproduction are unknown, and indeed, not all studies examining the relationship have consistently found adverse effects. Nutritional and other factors have been suggested but do not appear to be involved in patients with silent CD. [10].

More compelling evidence of the role of $\mathrm{CD}$ in unexplained infertility may be suggested by a positive response to therapy in those with yet-untreated diseases. There are several reports of successful pregnancy after adoption of a GFD, but the number of $\mathrm{CD}$ cases detected by screening has been too small and the concomitant use of other effective interventions limits conclusions about causality [9]. Testing of serum IgA anti-tissue transglutaminase antibodies is recommended initially in those without IgA deficiency, because it is highly sensitive and specific [1]. IgA anti-endomysial antibodies are nearly $100 \%$ specific but may only be used to confirm diagnosis, because they may be seen in other autoimmune disorders. Antigliadin antibody testing, once recommended for screening, lacks the sensitivity and specificity for use as a screening test in reproductive-aged patients [11].

Women having unexplained infertility, recurrent abortions, stillbirths or intrauterine growth retardation could have subclinical $\mathrm{CD}$, which can be detected by serological screening tests. Improved diagnostic tools and greater access to screening have led to more latent or subclinical CD. Untreated CD may lead to more reproductive problems if those patients don't follow a GFD. Until now, there have been very few studies regarding the effect of latent $\mathrm{CD}$ on reproductive performance .

Based on IgA tTG antibody titers, latent CD was 5.43 times more common in the group with recurrent spontaneous abortion than in the control group. The rate of latent $\mathrm{CD}$ in the group with stillbirth was 4.61 times greater than the control group. The rate for the group with intrauterine growth restriction was 7.75 times greater than the control subjects, while the rate for those with unexplained infertility was 4.51 times higher than the control.

The researchers also found that women with positive blood screens showed higher rates of previous early births, low-weight births and cesarean sections than the seronegative subjects did. Researchers admitted that not every study showed a clear reduction in fertility, but a number did show a higher risk of adverse pregnancy outcomes for women with latent CD. Spotting the CD and treating it with a gluten free diet may reduce these associated risks.

An increased incidence of reproductive problems, including infertility, miscarriage, low birth weight newborns and shorter duration of breast-feeding, is known to exist in women with coeliac diseases; some of these conditions are improved by a GFD. Screening for coeliac disease should be part of the diagnostic work-up of infertile women, particularly when no apparent cause can be ascertained after the standard evaluation [12].

\section{Experimental}

This study was planned to detect the percentage of aborted women having non-classical coeliac diseases 
with or without Toxoplasma infection. By using 125 pregnant women with single or multiple abortion from different private clinics and hospitals in Babylon province, as well as 30 age-matched women as the control group which included 15 pregnant women without history of abortion and 15 apparently healthy women without pregnancy. All studied cases had previous diagnosis by specialists as aborted women; most of them had unexplained (idiopathic) causes. Other causes of abortion were excluded such as infection with CMV, Rubella and anti-Phospholipid syndrome. For all patients and control, screening test for anti-Toxoplasma antibody (IgM and IgG) and AtTg IgA antibody level were done by using the manual principle and procedure of Elabsience company of ELISA technique in the laboratory of Babylon GIT Center during the period between August 2015 and June 2016. Statistical analysis was done by using ChiSquare and one way ANOVA tests in SPSS statistical program.

\section{Results and Discussion}

By using basic standard ELISA technique to determine AtTg IgA, anti-Toxoplasma IgG and IgM for all patients and control, the results were revealed as illustrated in Figs. 1-3. Aborted women had a higher level of both AtTg IgA and anti-Toxo IgG antibodies than the control group, with a highly significant $(\mathrm{P}<$ 0.01 ) increase, while IgM had a higher level but no significant increase. This result suggested that there was a relationship between aborted women having Toxoplasma infection and AtTg antibodies, which might indicate there was a latent (asymptomatic) coeliac disease associated with pregnancy leading to spontaous abortion. The result was consistent with another finding that women having poor reproductive performance had subclinical CD. The serology for CD

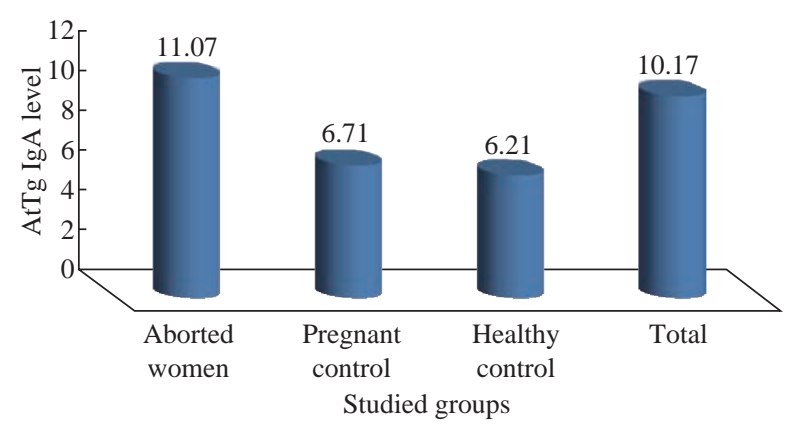

Fig. 1 The concentration of AtTg IgA level among all patients and control.

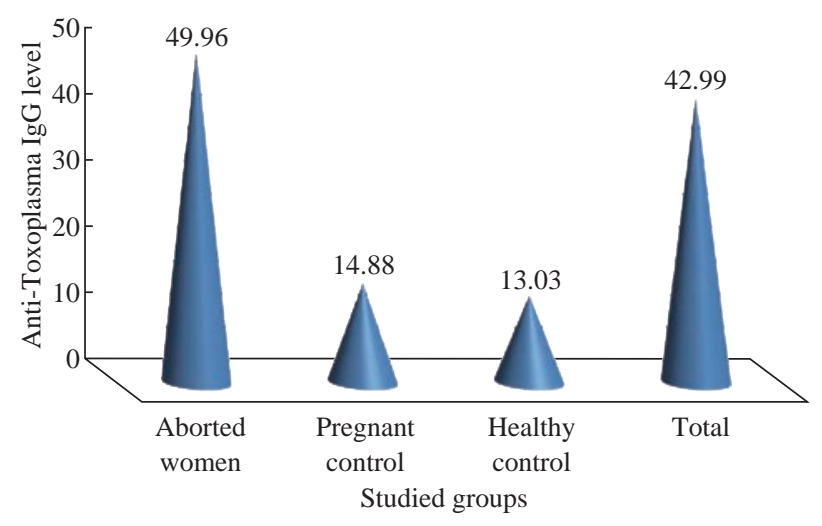

Fig. 2 The concentration of anti-Toxoplasma IgG level among all patients and control.

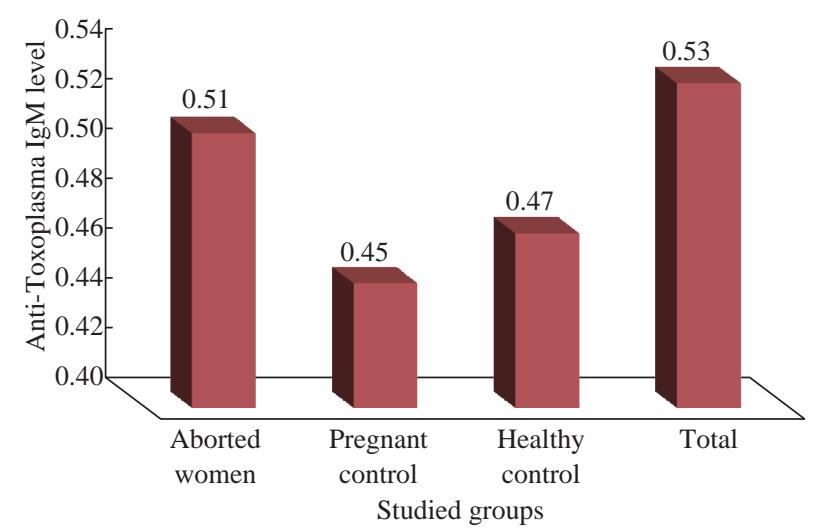

Fig. 3 The concentration of anti-Toxoplasma IgM level among all patients and control.

can be considered in idiopathic cases [10].

Elevated anti-IgM titers are often absent in immunocompromised patients. In addition, elevated IgM can persist from an acute infection that may have occurred as long ago as one year. IgG is only indicative of previous exposure to Toxoplasma (recent or past). A single positive Toxoplasma IgG result should not be used to diagnose recent infections [1].

Table 1 shows the highly significant increase of AtTg level in aborted women in comparison to the control group. There was no difference of AtTg level in pregnant women having anti-Toxo $\operatorname{IgG}$ antibody in comparison to the pregnant with negative $\operatorname{IgG}$ group. In addition, there was a highly significant increase in anti-Toxo IgG level among the infected group than the non-infected and the control groups. However, there was no significant result of anti-Toxo IgM antibody for all patients and control groups. This result suggested that AtTg level increased in pregnancy and might indicate the hidden reason of abortion rather than other causes. In $43 \%$ women after 6-8 months of strict adherence to a GFD stopped to have amenorrhea and 
Table 1 The relationship of anti-Toxoplasma IgG antibody and AtTg level among patients and control

\begin{tabular}{|c|c|c|c|c|c|}
\hline \multicolumn{2}{|c|}{ Groups } & \multirow{2}{*}{$\frac{\mathrm{N}}{55}$} & \multirow{2}{*}{$\frac{\operatorname{AtTg} \text { mean } \pm S D}{11.33 \pm 3.46}$} & \multirow{2}{*}{$\frac{\mathrm{IgG} \text { mean } \pm \mathrm{SD}}{94.46 \pm 15.82}$} & \multirow{2}{*}{$\frac{\operatorname{IgM} \text { mean } \pm \mathrm{SD}}{0.55 \pm 0.27}$} \\
\hline & $\mathrm{IgG}+$ & & & & \\
\hline & $\mathrm{IgG}^{-}$ & 70 & $10.86 \pm 3.23$ & $14.99 \pm 3.46$ & $0.57 \pm 0.15$ \\
\hline \multirow[t]{3}{*}{ Anti-Toxo IgG antibody } & Pregnant control & 15 & $6.71 \pm 2.61$ & $14.88 \pm 4.06$ & $0.48 \pm 0.18$ \\
\hline & Healthy control & 15 & $6.21 \pm 2.73$ & $13.04 \pm 3.86$ & $0.45 \pm 0.21$ \\
\hline & Total & 155 & $10.17 \pm 2.06$ & $42.99 \pm 15.44$ & $0.47 \pm 0.18$ \\
\hline \multicolumn{3}{|c|}{$P$ value of F-test and significance } & $0.002 \mathrm{HS}$ & $0.000 \mathrm{HS}$ & $0.374 \mathrm{NS}$ \\
\hline
\end{tabular}

IgG+: Pregnant with positive $\mathrm{IgG}$; IgG-: Pregnant with negative IgG

regular menses appeared. Three women of childbearing age strictly abided to the GFD and had a history of repeated spontaneous abortion during the year managed to get pregnant and give birth to healthy fullterm babies. Reproductive disorders are significantly more likely to occur in women with $\mathrm{CD}$ than in women with functional bowel diseases [3].

Table 2 shows that the level of anti-Toxo IgG antibody in the group of patients with weak or equivocal level of AtTg was higher than the negative, positive and control groups. This result shows that many patients with chronic Toxoplasma infection had a high risk of being infected with asymptomatic coeliac disease (non-classical type), and that they were a higher risk of repeated abortions than the other groups with a low level of AtTg.

Table 3 shows there was a highly significant increase in anti-Toxoplasma IgM level among the patients with positive results in comparison to patients with negative results and the control group. The AtTg-IgA level was higher among patient having negative IgM result, which might suggest that aborted women were predisposed to be infected with latent coeliac disaeses regardless of Toxoplasma infection. IgM antibodies, however, elevated anti-IgM titers are often absent in immunocompromised patients. In addition, elevated IgM can persist from an acute infection that may have occurred as long as one year ago [1].

Table 4 shows that the higher percentage of aborted women were distributed in age range of 25-24 years. This age range also shows a higher level of AtTg IgA in comparison to other age groups and the control group. This result indicates that young women are more susceptible to abortion than other age groups either with Toxoplasma infection or idiopathic abortion, due to their infection with the latent coeliac disease.

Most abortions are carried out for women between the ages of 18 and 29. Although abortion numbers are largely concentrated within the middle of the reproductive life-span of age 15 to 44 , a relatively small number of women at both ends of this spectrum require abortions. Over the recent decades, a great deal of attention has been paid to "teenage" pregnancy and abortion rates. It should first be noted that the category

Table 2 The level of anti-AtTg among all patients and control groups in relation to anti-Toxo antibody levels

\begin{tabular}{cccccc}
\hline \multicolumn{2}{c}{ Groups } & N & AtTg Mean \pm SD & IgG Mean \pm SD & IgM Mean \pm SD \\
\hline \multirow{2}{*}{ AtTg level } & -ve & 88 & $8.15 \pm 1.96$ & $42.04 \pm 18.46$ & $0.54 \pm 0.16$ \\
& Weak & 22 & $12.59 \pm 1.99$ & $85.97 \pm 19.35$ & $0.56 \pm 0.14$ \\
& Pregnant control & 15 & $25.97 \pm 5.27$ & $43.58 \pm 19.57$ & $0.48 \pm 0.18$ \\
& Healthy control & 15 & $6.71 \pm 2.61$ & $14.88 \pm 4.01$ & $0.45 \pm 0.21$ \\
& Total & 15 & $6.21 \pm 2.73$ & $13.04 \pm 6.86$ & $0.47 \pm 0.18$ \\
& & 155 & $10.17 \pm 6.06$ & $42.98 \pm 15.44$ & $0.54 \pm 0.27$ \\
\hline
\end{tabular}

-ve: Negative; +ve: Positive

-ve AtTg < 10; weak: $10-15$; +ve AtTg > 15 
Table 3 The relationship between anti-Toxoplasma IgM antibody and AtTg level among patients and control

\begin{tabular}{|c|c|c|c|c|c|}
\hline \multicolumn{2}{|c|}{ Groups } & \multirow{2}{*}{$\frac{N}{5}$} & \multirow{2}{*}{$\begin{array}{c}\text { AtTg Mean } \pm \text { SD } \\
8.46 \pm 2.13\end{array}$} & \multirow{2}{*}{$\begin{array}{c}\text { IgG } \text { Mean } \pm \text { SD } \\
18.46 \pm 6.82\end{array}$} & \multirow{2}{*}{$\begin{array}{c}\operatorname{IgM} \text { Mean } \pm \mathrm{SD} \\
2.32 \pm 0.80\end{array}$} \\
\hline & $+\mathrm{ve}$ & & & & \\
\hline & - ve & 120 & $11.18 \pm 3.41$ & $14.99 \pm 3.46$ & $0.47 \pm 0.19$ \\
\hline \multirow[t]{3}{*}{ Anti-Toxo IgM antibody } & Pregnant control & 15 & $6.71 \pm 2.61$ & $14.88 \pm 4.01$ & $0.45 \pm 0.22$ \\
\hline & Healthy control & 15 & $6.21 \pm 2.73$ & $13.03 \pm 6.86$ & $0.47 \pm 0.19$ \\
\hline & Total & 155 & $10.17 \pm 2.06$ & $42.99 \pm 15.43$ & $0.56 \pm 0.41$ \\
\hline \multicolumn{3}{|c|}{$P$ value of F-test and significance } & $0.001 \mathrm{HS}$ & $0.086 \mathrm{NS}$ & $0.009 \mathrm{HS}$ \\
\hline
\end{tabular}

-ve: Negative; +ve: Positive

Table 4 Age group distribution and AtTg IgA level among all patient groups

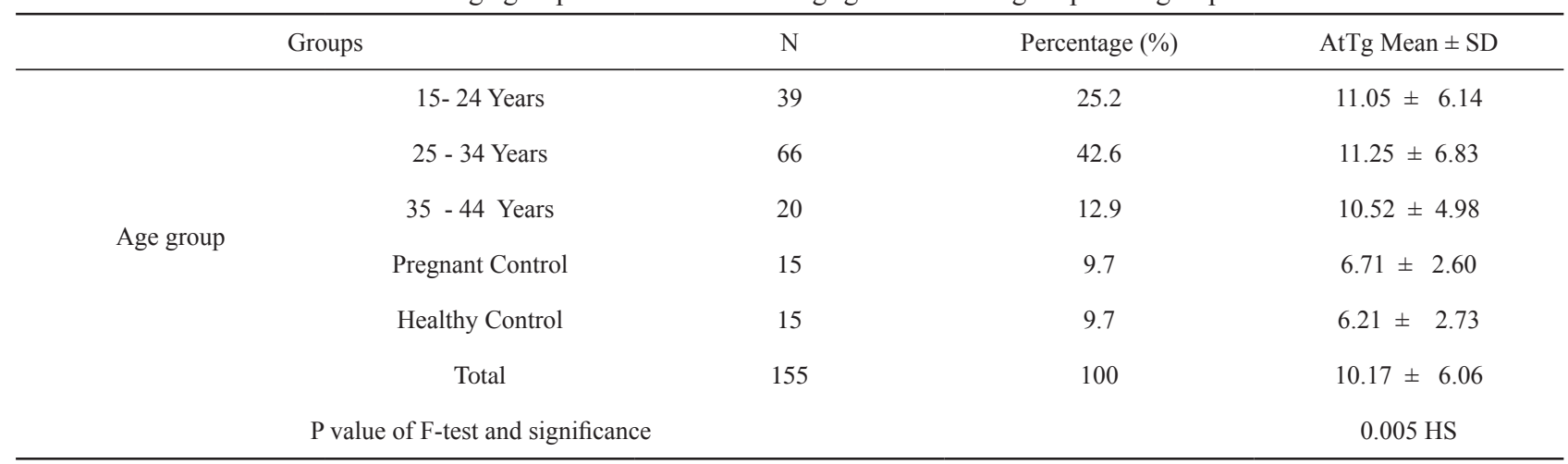

Table 5 Abortion time in association with AtTg IgA levels among all patients groups

\begin{tabular}{|c|c|c|c|c|}
\hline & & $\mathrm{N}$ & Percent $(\%)$ & $\operatorname{AtTg}$ Mean \pm SD \\
\hline \multirow{7}{*}{ Abortion time } & First trimester & 65 & 41.9 & $11.04 \pm 6.49$ \\
\hline & Second trimester & 24 & 15.5 & $11.06 \pm 6.05$ \\
\hline & Third trimester & 36 & 23.2 & $11.11 \pm 6.33$ \\
\hline & Pregnant control & 15 & 9.7 & $6.71 \pm 2.60$ \\
\hline & Healthy control & 15 & 9.7 & $6.21 \pm 2.73$ \\
\hline & Total & 155 & 100 & $10.17 \pm 6.06$ \\
\hline & $P$ value of F-test and significance & & & $0.006 \mathrm{HS}$ \\
\hline
\end{tabular}

"teenage" does not distinguish between girls who are pregnant at the age of 14 and those at the age of 19. The standardized average (mean) age of mother rose to 29.5 years in 2010 , compared with 29.4 years in 2009 and 28.5 years in 2000 [13].

According to Table 5, abortions during the first trimester had a higher percentage of $41.9 \%$ and a higher AtTg IgA level in comparison to the second and third trimesters as well as the control groups. This result might suggest that abortion was more distributed in the first trimester than the other periods, so the follow-up and special maintenance was recommended in such time rather than others. CD also has been suggested as a contributor to recurrent pregnancy loss. In a small series, $8 \%$ of affected patients had positive serology and the diagnosis of CD was confirmed by biopsy in $88 \%$. [14]. Women with undiagnosed CD had the chance of miscarriage 9 times higher than those who were diagnosed and had gone gluten free. These miscarriages can happen at any time in pregnancy and sadly, are often late-term miscarriages. There is a suspicion that they may be due to malnourishment of the fetus [16].

Abortion, low birth weight of baby and duration of breast feeding were not significantly related to the severity of $\mathrm{CD}$ among untreated women. Results indicated that the gluten-free diet reduced the relative risk of abortion by 9.18 times. reduced the number of low birth weight babies from $29.4 \%$ to zero ( $p<0.05$ ), and increased the duration of breast feeding 2.38 times 
$(\mathrm{p}<0.10)$. The high incidences of abortion, low birth weight babies and short breast-feeding periods were effectively corrected by gluten-free diet in women with CD [16].

The result in Table 6 shows that $52.3 \%$ of patients had complete abortion without any live children, and that $28.4 \%$ of patients had either single or more live children. Both groups of patients presented a higher level of AtTg IgA antibody than the control groups. This result reveals the chance of an infected woman giving live birth was very poor, especially when the patient had latent or asymptomatic infection with coeliac disease.

The result in Table 7 shows that $34.8 \%$ of aborted women had single abortion, and the AtTg IgA antibody showed a high level in the single abortion and the other

Table 6 Abortion with children in association with AtTg IgA level among all patient groups

\begin{tabular}{lcccc}
\hline & & $\mathrm{N}$ & Percentage (\%) & AtTg mean \pm SD \\
\hline \multirow{4}{*}{ Abortion with children } & No children & 81 & 52.3 & $11.0406 \pm 6.83562$ \\
& With children & 44 & 28.4 & $11.1098 \pm 5.28134$ \\
& Pregnant control & 15 & 9.7 & $6.7067 \pm 2.60370$ \\
& Healthy control & 15 & 9.7 & $6.2060 \pm 2.72684$ \\
& Total & 155 & & $10.1730 \pm 6.05833$ \\
& P value of F-test and significance & & & $0.002 \mathrm{HS}$ \\
\hline
\end{tabular}

Table 7 Relationship between abortion and AtTg IgA level among all patient groups

\begin{tabular}{ccccc}
\hline & & $\mathrm{N}$ & Percentage (\%) & AtTg mean \pm SD \\
\hline & Single abortion & 54 & 34.8 & $11.6639 \pm 7.28244$ \\
Dobortion number abortion & 38 & 24.5 & $10.0963 \pm 5.29672$ \\
& Multiple abortion & 33 & 21.3 & $11.2003 \pm 5.68194$ \\
& Pregnant control & 15 & 9.7 & $6.7067 \pm 2.60370$ \\
& Healthy control & 15 & 100 & $6.2060 \pm 2.72684$ \\
& Total & 155 & & $10.1730 \pm 6.05833$ \\
\hline
\end{tabular}

Table 8 Correlation between AtTg IgA level and other studied parameters among all patient groups

\begin{tabular}{|c|c|c|c|c|c|c|c|c|}
\hline & & AtTg level & Toxo IgG & Age & $\begin{array}{c}\text { Abortion } \\
\text { duration }\end{array}$ & Toxo IgM & With children & $\begin{array}{c}\text { Abortion } \\
\text { number }\end{array}$ \\
\hline AtTg level & $\begin{array}{c}\text { Pearson } \\
\text { correlation, } \\
\text { Sig. (2-tailed) }\end{array}$ & 1 & & & & & & \\
\hline \multirow{2}{*}{ Toxo IgG } & $\begin{array}{c}\text { Pearson } \\
\text { correlation, }\end{array}$ & 0.114 & 1 & & & & & \\
\hline & Sig. (2-tailed) & 0.156 & & & & & & \\
\hline \multirow{2}{*}{ Age } & $\begin{array}{c}\text { Pearson } \\
\text { correlation, }\end{array}$ & $-0.269-* *$ & $-0.219-* *$ & 1 & & & & \\
\hline & Sig. (2-tailed) & 0.001 & 0.006 & & & & & \\
\hline \multirow{2}{*}{$\begin{array}{l}\text { Abortion } \\
\text { duration }\end{array}$} & $\begin{array}{l}\text { Pearson } \\
\text { correlation, }\end{array}$ & $-0.241-* *$ & $-0.253-* *$ & $0.760 * *$ & 1 & & & \\
\hline & Sig. (2-tailed) & 0.002 & 0.001 & 0.000 & & & & \\
\hline \multirow{2}{*}{ Toxo IgM } & $\begin{array}{c}\text { Pearson } \\
\text { correlation, }\end{array}$ & $-0.032-$ & $-0.046-$ & $-0.044-$ & $-0.009-$ & 1 & & \\
\hline & Sig. (2-tailed) & 0.697 & 0.568 & 0.587 & 0.916 & & & \\
\hline \multirow{2}{*}{ With children } & $\begin{array}{l}\text { Pearson } \\
\text { correlation, }\end{array}$ & $-0.264-* *$ & $-0.177-*$ & $0.705^{* *}$ & $0.684 * *$ & $-0.015-$ & 1 & \\
\hline & Sig. (2-tailed) & 0.001 & 0.027 & 0.000 & 0.000 & 0.858 & & \\
\hline \multirow{2}{*}{$\begin{array}{c}\text { Abortion } \\
\text { number }\end{array}$} & $\begin{array}{c}\text { Pearson } \\
\text { correlation, }\end{array}$ & $-0.270-* *$ & $-0.159-*$ & $0.772 * *$ & $0.730 * *$ & 0.001 & $0.693 * *$ & 1 \\
\hline & Sig. (2-tailed) & 0.001 & 0.048 & 0.000 & 0.000 & 0.993 & 0.000 & \\
\hline
\end{tabular}


groups of patients (double and multiple abortions) in comparison to the control groups. An analysis of the cases that emerged from this study and those reported in the literature show that the tests to identify the CD should extend to the population with a risk of developing this disease. These subjects should include those with a family history or clinical symptoms, in particular women with a history of multiple abortions. In these cases, there are grounds for suspecting an atypical form of CD [15-19].

Table 8 shows a direct correlation between AtTg $\operatorname{IgA}$ and anti-Toxoplasma IgG antibody, and an indirect or negative correlation with anti-Toxoplasma IgM antibody although there was no statistically significant result. AtTg IgA had a significant negative correlation with age group, duration of abortion and abortion number. This result might suggest that AtTg IgA spontaneously increased with time in relation to patient ages as well as the specific condition of aborted women such as time and number of abortion.

\section{Conclusions}

There is a relationship between aborted women having Toxoplasma infection with AtTg antibody, which suggested there was a latent (asymptomatic) coeliac disease associated with pregnancy, leading to the spontaneous abortion. IgG was only indicative of previous exposures to Toxoplasma (recent or past). A single positive Toxoplasma IgG result should not be used to diagnose recent infections. Women aged 24-35 years were more susceptible to abortions than the other age groups, mostly at the first trimester of pregnancy. There was a direct correlation between AtTg IgA and anti-Toxoplasma IgG antibody. AtTg IgA spontaneously increased with time in relation to patient ages, as well as with specific conditions of aborted women such as time and number of abortion.

\section{Conflict of Interests}

The authors declare that no competing interest exists.

\section{References}

[1] A.M. Tenter, A.R. Heckeroth, L.M. Weiss, Toxoplasma gondii: From animals to humans. Int. J. Parasitol., 2000,
30(12-13): 1217-1224.

[2] J.L. Jones, D. Kruszon-Moran, K. Sanders-Lewis, Toxoplasma gondii infection in the United States, 19992004, decline from the prior decade. Am. J. Trop. Med. Hyg., 2007, 77(3): 405-411.

[3] S.V. Bykova, E.A. Sabel'nikova, and A.I. Parfenov, Reproductive disorders in women with celiac disease. Effect of the etiotropic therapy. Gastroenterologiia. Experimental \& Clinical Gastroenterology, 2011, (3): 1218.

[4] S. Guandalini, A. Assiri, Celiac disease: a review. JAMA Pediatr., 2014, 168: 272-285.

[5] A. Fasano, C. Catassi, Clinical practice. Celiac disease. $N$. Engl. J. Med., 2012, 20: 2419-2426.

[6] M, Stephanie, C. Christina, and J. Lindenmeyer, Increased rates of pregnancy complications in women with celiac disease. Ann. Gastroenterol., 2015, 28(2): 236-240.

[7] A. Kumar, M. Meena, and N. Begum, Latent celiac disease in reproductive performance of women. 2011, 95(3): 922-927.

[8] P. Craig, M.D. Griebel, and M.D.J. Halvorsen, Management of spontaneous abortion, University of Illinois, College of Medicine at Peoria, Peoria, Illinois. Am Fam Physician., 2005, 72(7): 1243-1250.

[9] R. Nenna, M. Mennini, and L. Petrarca, Immediate effect on fertility of a gluten-free diet in women with untreated coeliac disease. Gut., 2011, 60(7): 1023-1024.

[10] A.S. Khashan, T.B. Henriksen, and P.B. Mortensen, The impact of maternal celiac disease on birthweight and preterm birth: A Danish population-based cohort study. Hum Reprod., 2010, 25(2): 528-534.

[11] R. Daniel, M.D. Christie, Reproductive effects of celiac disease. S.J. Ory MD, D.R. Christie MD, Contemporary OB/GYNObstetrics-Gynecology \& Women's Health. 2013.

[12] J. Adams, Latent celiac disease can increase reproductive problems. World J. Gastroenterol., 2010, 16(46): 58105814.

[13] L. Hallgarten, Statistics briefing (4): Age, marital status, previous children, and ethnicity. Abortion Review, 2 May 2012

[14] A. Gasbarrini, E.S. Torre, and C. Trivellini, Recurrent spontaneous abortion and intrauterine fetal growth retardation as symptoms of coeliac disease. Lancet, 2000, 356(9227): 399-400.

[15] F. Foschi, F. Diani, E. Zardini, et al., Celiac disease and spontaneous abortion. Minerva Ginecol., 2002, 54(2): 151-159.

[16] C. Ciacci, M. Cirillo, G. Auriemma, et al., Celiac disease and pregnancy outcome. Am. J. Gastroenterol., 1996, 91(4): 718-722.

[17] Mel, Celiac disease and it's connection to miscarriage and infertility. Blogher Health Network, 2015.

[18] R.R Al-Araji, M.S. Mashkour, and E.A.J. Al-Mulla, Spectrophotometric determination of vitamin folic acid B9 in some drugs using 1, 2-naphthoquine-4-sulphonate (NQS). Nano Biomed. Eng 2017, 9 (3), 208-213.

[19] M.M. Radhi, H.A.T. Al-Shimmari, E.A.J. Al-Mulla, et al., New voltammetric study of $\mathrm{MgCl}_{2}$ as alternative contrast media in MRI molecular imaging. Nano Biomed. Eng., 2017, 9(2): 152-161.

Copyright $($ Shukryia Shadhan Chyad. This is an openaccess article distributed under the terms of the Creative Commons Attribution License, which permits unrestricted use, distribution, and reproduction in any medium, provided the original author and source are credited. 